\title{
REBOA or Preperitoneal Packing in Patients with Pelvic Fractures: Why Not Both?
}

\author{
Maya Paran ${ }^{1}$, Boris Kessel ${ }^{1}$ and Eyal Hashavia ${ }^{2}$ \\ 'Surgical Division, Hillel Yaffe Medical Center, affiliated with Rappaport Medical School, Technion, Haifa, Israel \\ ${ }^{2}$ Trauma Unit, General Surgery Division, Tel Aviv Sourasky Medical Center, Israel
}

A non-Shakespearean scenario of a hemodynamically unstable patient with pelvic fractures is a challenge for any highly professional trauma team. The treatment paradigm of this life-threatening condition has changed multiple times during the past decades, depending on the institution's facilities and teams' availability. In the 1980s and early 1990s, the standard approach consisted of exploratory laparotomy and internal packing with or without bilateral internal iliac ligation [1]. With passing years, accumulated experience has demonstrated that such procedures are ineffective and should therefore be abandoned when treating patients with isolated pelvic fractures and no concomitant intra-abdominal injury requiring laparotomy. The accepted treatment shifted to other surgical procedures, such as different types of external pelvic ring fixation, which aim to decrease the pelvic volume and create local tamponade [2]. These techniques became considered as the standard care until several studies reported that hemodynamic instability, in an adequately resuscitated patient with pelvic fractures, is a marker of arterial injury. Hence, pelvic angioembolization has become the preferred approach [2]. However, safe invasive radiology techniques require adequate physiological parameters to allow a transfer to the angiography suite and, occasionally, time-consuming procedures [3].

During the late 1990s and early 2000s, pelvic preperitoneal packing (PPP) was introduced for primary stabilization of patients with pelvic fractures [4-6]. This relatively simple and quick technique, which can be

\footnotetext{
Corresponding author:

Dr. Maya Paran, Hashalom St. 2, Hadera, Israel. POB 169, 3846201. Email: Paran.maya@gmail.com

(c) 2021 CC BY 4.0 - in cooperation with Depts. of Cardiothoracic/ Vascular Surgery, General Surgery and Anesthesia, Örebro University Hospital and Örebro University, Sweden
}

performed in the emergency room, achieves a rapid blood pressure improvement in most cases $[7,8]$ and has thus become favored worldwide [9]. Additionally, depending on the source of bleeding, some authors reported no need to follow up with angio-embolization after the packing $[10,11]$. Although angio-embolization is the treatment of choice for arterial bleeding, it does not address the more prevalent venous bleeding, for which PPP is an important treatment option [12]. For example, Burlew et al. [10] have reported arterial bleeding to be present in only $13 \%$ of patients with pelvic fractures, making the need for angio-embolization very limited. Nevertheless, despite gaining popularity, PPP has been reported to be associated with several disadvantages. The main concern is with regard to the next appropriate step when it does not work. To date, when treating an unstable blunt trauma patient with a positive FAST and an unstable pelvic ring, the question remains: Should explorative laparotomy or PPP be performed first? In the presence of an open abdomen, the efficacy of the packing markedly decreases. In addition, performing PPP prior to laparotomy possibly limits the necessary abdominal exposure. Other disadvantages of PPP include increased morbidity associated with essential de-packing, closure of wounds, increased infection rates [13], etc.

Surprisingly, although REBOA was first approved for hemorrhage control in patients with pelvic fractures [14-16] and is mentioned in various guidelines/recommendations for pelvic fracture management [17-19], only a few studies have compared REBOA with PPP in these patients. Mikdad et al. [20] have published a retrospective analysis of 204 blunt trauma patients, of which 102 were treated with PPP and 102 matched patients who were treated with REBOA placement. No significant differences in blood transfusion volume, length of hospital stay or rates of major complications were reported. Time to intervention was shorter in those patients treated with REBOA. However, REBOA was found to be associated with higher rates of 24-h mortality and in-hospital mortality. 
Another study by Duchesne et al. [21] that investigated the outcome of different methods for hemorrhage control included 24 patients who underwent PPP and 7 who were treated with REBOA. No differences between the two groups were found with regard to median injury severity score (ISS), rates of head abbreviated injury scale (AIS) $\geq 3$, chest AIS $\geq 3$, extremity AIS $\geq 3$, median Glasgow coma scale, heart rate, and systolic blood pressure. The authors have found PPP to be associated with mortality rates of $58 \%$, whereas REBOA was associated with a mortality rate of $86 \%(P<0.001)$. Median length of hospital stay was 16 (1-33) days for PPP and $1(1-2)$ days for REBOA $(P=0.017)$.

In the most recent published study, Asmar et al. [22] also compared these two techniques in the management of hemodynamically unstable patients with pelvic fractures. This study included 156 patients, of which 52 were treated with PPP, 52 with REBOA, and another group of 52 with both PPP and REBOA. The authors found that both 24-h mortality and in-hospital mortality were highest in the patients who underwent both PPP and REBOA. However, these mortality rates were lowest in the group of patients who were treated with REBOA alone, even when compared with the group treated with both REBOA and PPP. Moreover, time to laparotomy and/or angioembolization was also shorter in the REBOA-only group.

To the best of our knowledge, the above-mentioned study, was a pioneer evaluation of the feasibility of a combination of REBOA and PPP. Unfortunately, this article doesn't include the crucial details of such combined approach. When reading through this paper, two questions arise: Which was performed first, the PPP or REBOA? and Is it feasible to perform both procedures simultaneously? There are several aspects that we believe should be further discussed. From a technical point of view, simultaneous performance of these two procedures is feasible. Normally, the blood pressure in the iliac artery is nearly similar to the aortic blood pressure. Therefore, PPP, even when performed after zone-III balloon inflation, cannot occlude arterial blood flow. Furthermore, an arterial access achieved via the groin approach does not decrease the efficacy of PPP. Certainly, the ability to perform these techniques relies on the immediate availability of properly trained teams.

In summary, there is no single appropriate treatment for patients with unstable pelvic fractures. The choice of treatment must also rely on appropriate teams' availability and the specific medical center's resources. Most patients with unstable pelvic fractures respond adequately to PPP, allowing for follow-up investigation and pelvic angioembolization. In the small group of PPP non-responders, the mortality rates remain high. REBOA is a temporary bridging technique for blood pressure stabilization, which isn't always efficient. Simultaneous use of REBOA and PPP or preparedness for placement of REBOA with achievement of immediate access may provide safe patient transfer to a hybrid room where any endovascular treatment could be maximally utilized. In this editorial, we call for future animal and human studies to investigate and better define the indications, proper timing and feasibility of a truly hybrid approach, combining both PPP and REBOA.

\section{Ethics Statement}

(1) All the authors mentioned in the manuscript have agreed to authorship, read and approved the manuscript, and given consent for submission and subsequent publication of the manuscript.

(2) The authors declare that they have read and abided by the JEVTM statement of ethical standards including rules of informed consent and ethical committee approval as stated in the article.

\section{Conflicts of Interest}

The authors declare that they have no conflicts of interest.

\section{Funding}

The authors received no financial support for the research, authorship, and/or publication of this article.

\section{Author Contributions}

All authors have contributed to the writing and editing of this manuscript.

\section{REFERENCES}

[1] Riska EB, von Bonsdorff H, Hakkinen S, Jaroma H, Kiviluoto O, Paavilainen T. Operative control of massive haemorrhage in comminuted pelvic fractures. Int Orthop. 1979;3(2):141-4.

[2] Flint L, Cryer HG. Pelvic fracture: the last 50 years. J Trauma. 2010;69(3):483-8.

[3] Tesoriero RB, Bruns BR, Narayan M, Dubose J, Guliani SS, Brenner ML, Boswell S, Stein DM, Scalea TM. Angiographic embolization for hemorrhage following pelvic fracture: Is it "time" for a paradigm shift? J Trauma Acute Care Surg. 2017;82(1):18-26.

[4] Pohlemann T, Gansslen T, Bosch A, Tscherne U. The technique of packing for control of hemorrhage in complex pelvic fractures. Tech Orthop. 1994;9:267-70.

[5] Ertel W, Keel M, Eid K, Platz A, Trentz O. Control of severe hemorrhage using C-clamp and pelvic packing in multiply injured patients with pelvic ring disruption. J Orthop Trauma. 2001;15(7):468-74.

[6] Tötterman A, Madsen JE, Skaga NO, Røise O. Extraperitoneal pelvic packing: a salvage procedure to control massive traumatic pelvic hemorrhage. J Trauma. 2007;62(4):843-52.

[7] Jang JY, Shim H, Jung PY, Kim S, Bae KS. Preperitoneal pelvic packing in patients with hemodynamic instability due to severe pelvic fracture: early experience in a 
Korean trauma center. Scand J Trauma Resusc Emerg Med. 2016;24:3.

[8] Tai DKC, Li W-H, Lee K-Y, Cheng M, Lee K-B, Tang L-F, Lai AK-H, Ho H-F, Cheung M-T. Retroperitoneal pelvic packing in the management of hemodynamically unstable pelvic fractures: a level I trauma center experience. J Trauma. 2011;71:E79-E86.

[9] Cheng M, Cheung MT, Lee KY, Lee KB, Chan SC, Wu AC, Chow YF, Chang AM, Ho HF, Yau KK. Improvement in institutional protocols leads to decreased mortality in patients with haemodynamically unstable pelvic fractures. Emerg Med J. 2015;32(3):214-20.

[10] Burlew CC, Moore EE, Stahel PF, et al. Preperitoneal pelvic packing reduces mortality in patients with life-threatening hemorrhage due to unstable pelvic fractures. J Trauma Acute Care Surg. 2016; 82(2):233-42.

[11] Burlew CC, Moore EE, Smith WR, Johnson JL, Biffl WL, Barnett CC, Stahel PF. Preperitoneal pelvic packing/ external fixation with secondary angioembolization: optimal care for life-threatening hemorrhage from unstable pelvic fractures. J Am Coll Surg. 2011; 212:628-37.

[12] Huittinen VM, Slatis P. Postmortem angiography and dissection of the hypogastric artery in pelvic fractures. Surgery 1973;73:454-62.

[13] Cothren CC, Osborn PM, Moore EE, et al. Preperitonal pelvic packing for hemodynamically unstable pelvic fractures: a paradigm shift. J Trauma. 2007; 62:834-42.

[14] Martinelli T, et al. Intra-aortic balloon occlusion to salvage patients with life-threatening hemorrhagic shocks from pelvic fractures. J Trauma. 2010; 68: 942-8.

[15] Morrison JJ, et al. A systematic review of the use of resuscitative endovascular balloon occlusion of the aorta in the management of hemorrhagic shock. J Trauma Acute Care Surg. 2016;80:324-34.

[16] Coccolini F, Ceresoli M, McGreevy DT, Sadeghi M, Pirouzram A, Toivola A, Skoog P, Idoguchi K, Kon Y, Ishida T, Matsumura Y, Matsumoto J, Reva V, Maszkowski M, Fugazzola P, Tomasoni M, Cicuttin E, Ansaloni L, Zaghi C, Sibilla MG, Cremonini C, Bersztel A, Caragounis EC,
Falkenberg M, Handolin L, Oosthuizen G, Szarka E, Manchev V, Wannatoop T, Chang SW, Kessel B, Hebron D, Shaked G, Bala M, Ordoñez CA, Hibert-Carius P, Chiarugi M, Nilsson KF, Larzon T, Gamberini E, Agnoletti V, Catena F, Hörer TM. Aortic balloon occlusion (REBOA) in pelvic ring injuries: preliminary results of the ABO Trauma Registry. Updates Surg. 2020; 72(2):527-36.

[17] Coccolini F, Stahel PF, Montori G, et al. Pelvic trauma: WSES classification and guidelines. World J Emerg Surg. 2017;12:5.

[18] Shatz DV, Mitchell J. Western Trauma Association Critical Decisions in Trauma: management of pelvic fracture with hemodynamic instability -2016 updates. J Trauma Acute Care Surg 2016;81:1171-4.

[19] Magee GA, Fox CJ, Moore EE. Resuscitative endovascular balloon occlusion of the aorta in pelvic ring fractures: The Denver Health protocol. Injury. 2020;31. doi: 10.1016/j.injury.2020.01.044.

[20] Mikdad S, van Erp IAM, Moheb ME, Fawley J, Saillant N, King DR, Kaafarani HMA, Velmahos G, Mendoza AE. Pre-peritoneal pelvic packing for early hemorrhage control reduces mortality compared to resuscitative endovascular balloon occlusion of the aorta in severe blunt pelvic trauma patients: A nationwide analysis. Injury. 2020;51(8):1834-9.

[21] Duchesne J, Costantini TW, Khan M, Taub E, Rhee P, Morse B, Namias N, Schwarz A, Graves J, Kim DY, Howell E, Sperry J, Anto V, Winfield RD, Schreiber M, Behrens B, Martinez B, Raza S, Seamon M, Tatum D. The effect of hemorrhage control adjuncts on outcome in severe pelvic fracture: A multi-institutional study. J Trauma Acute Care Surg. 2019;87(1):117-24.

[22] Asmar S, Bible L, Chehab M, Tang A, Khurrum M, Douglas M, Castanon L, Kulvatunyou N, Joseph B. Resuscitative endovascular balloon occlusion of the aorta vs pre-peritoneal packing in patients with pelvic fracture. J Am Coll Surg. 2021;232(1): 17-26.e2. 